\title{
James Webb Space Telescope primary mirror integration: testing the multiwavelength interferometer on the test bed telescope
}

\author{
Gene Olczak*a and David J. Fischer ${ }^{\mathrm{a}}$, Mark Connolly ${ }^{\mathrm{a}}$, and Conrad Wells ${ }^{\mathrm{a}}$ \\ ${ }^{a}$ ITT Geospatial Systems, 1447 St. Paul Street, P.O. Box 60488, Rochester, NY 14606-0488
}

\begin{abstract}
The James Webb Space Telescope (JWST) integration includes a center of curvature test on its 18 primary mirror segment assemblies (PMSAs). This important test is the only ground test that will demonstrate the ability to align all 18 PMSAs. Using a multi-wavelength interferometer (MWIF) integrated to the test bed telescope (TBT), a one-sixth scale model of the JWST, we verify our ability to align and phase the 18 PMSAs. In this paper we will discuss data analysis and test results when using the MWIF to align the segments of the TBT in preparation for alignment of the JWST.
\end{abstract}

Keywords: Multiple-Wavelength, Interferometry, James Webb Space Telescope, JWST, Cryogenic, Vacuum, Optical, Metrology

*gene.olczak@itt.com; phone 1585 269-6102; itt.com

\section{INTRODUCTION}

The James Webb Space Telescope (JWST) is a three mirror anastigmat (TMA) where the eighteen primary mirror (PM) positions and radii are adjustable on orbit. However, there is no full aperture system test of the system during integration (the 6.6 meter diameter flat or collimator that would be required for this is prohibitively expensive). Thus, the only time the full PM is observed during testing is from the null lens at the PM center of curvature. To support un-ambiguous piston measurement between segments an all reflective null lens and multiple wavelength interferometer (MWIF) are used in the test.

The test bed telescope (TBT) is a one-sixth scale model of the JWST. The TBT does not have center of curvature test capability but it does provide full aperture test capability of the full system in double pass. This being the case, the TBT provides an excellent platform to develop and test the functionality of the MWIF hardware and software.

Prior to the TBT tests, the demonstrated performance of the MWIF has been shown to be quite good (Reference 1) but limited in scope. For example the target for piston measurements was limited to four large field segments with a few fixed steps. The test conditions were relatively benign, with no vibration or other noise, no actuation of the segments and relatively high surface quality. In contract, the TBT has 18 actuated segments of variable surface quality and is prone to vibration and drift (due to an aluminum primary mirror segment back plane).

Additional motivation for this test: The planned path-finder activities for Johnson Space Center (JSC) integration are insufficient for MWIF debug \& ITT software development. These activities only use two on the JWST PMSAs and so do not provide the full complexity of the JSC cryogenic test. Without the MWIF TBT test the MWIF debug \& ITT software development would occur during the JSC cryogenic test. Due to the cost associated with JSC cryogenic test time this would be very inefficient.

The MWIF integration to the TBT is illustrated in Figure 1 a) and b). The MWIF is inserted into the TBT using an offthe-shelf afocal relay. A pair of "figure four" configured fold mirrors provides alignment capability. The insertion optics penetrate a thermal shroud that encapsulates the TBT. 


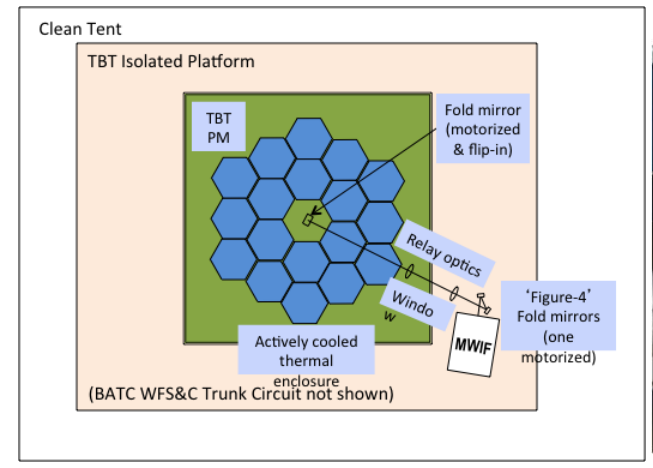

(a) TBT Layout

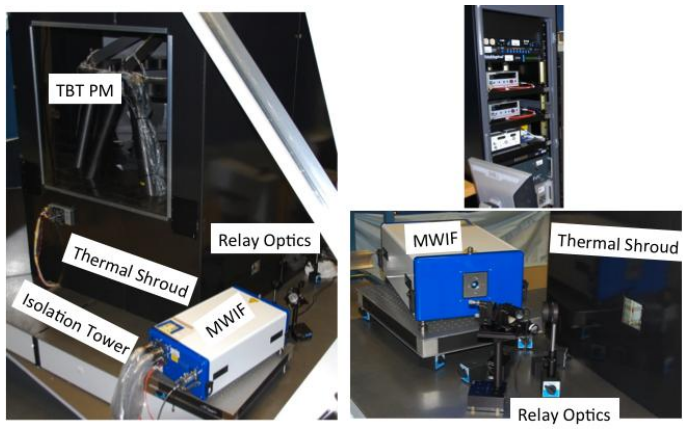

(b) Hardware Photographs

Figure 1. MWIF integration at TBT.

\section{DATA FLOW OVERVIEW}

A simplified flow chart of the ITT's Segmented Aperture Software Suite (SASS) processing of the TBT data is in Figure 2. All data analysis steps are completed in MATLAB as either m-code or compiled mex files. All MWIF image manipulation occurs in the sample space of the instrument with respect to pupil map position. Prior to evaluating data, the TBT PMSA optical sensitivities must be mapped into the MWIF image domain. Thus computation of mirror alignment (pose) occurs without computing the distortion correction of each data image. This allows for efficient in-theloop estimation of mirror pose so that the statistical spread of each mirror alignment degree-of-freedom (DOF) is minimized in a measurement sequence. Two sets of sensitivities are employed: global sensitivities have a common geometric reference for all segments (convenient for tracking global drift), while local sensitivities are used to communicate requests for PMSA pose changes to the PMSA control software. The development of major components of the SASS is outlined in Figure 3. Some of the components of SASS are typical of phase shifting interferometry (phase computation, 2D unwrapping, modulation threshold, etc.). In the following sections aspects of SASS unique to multiple wavelength operation and segmented system alignment are discussed. 


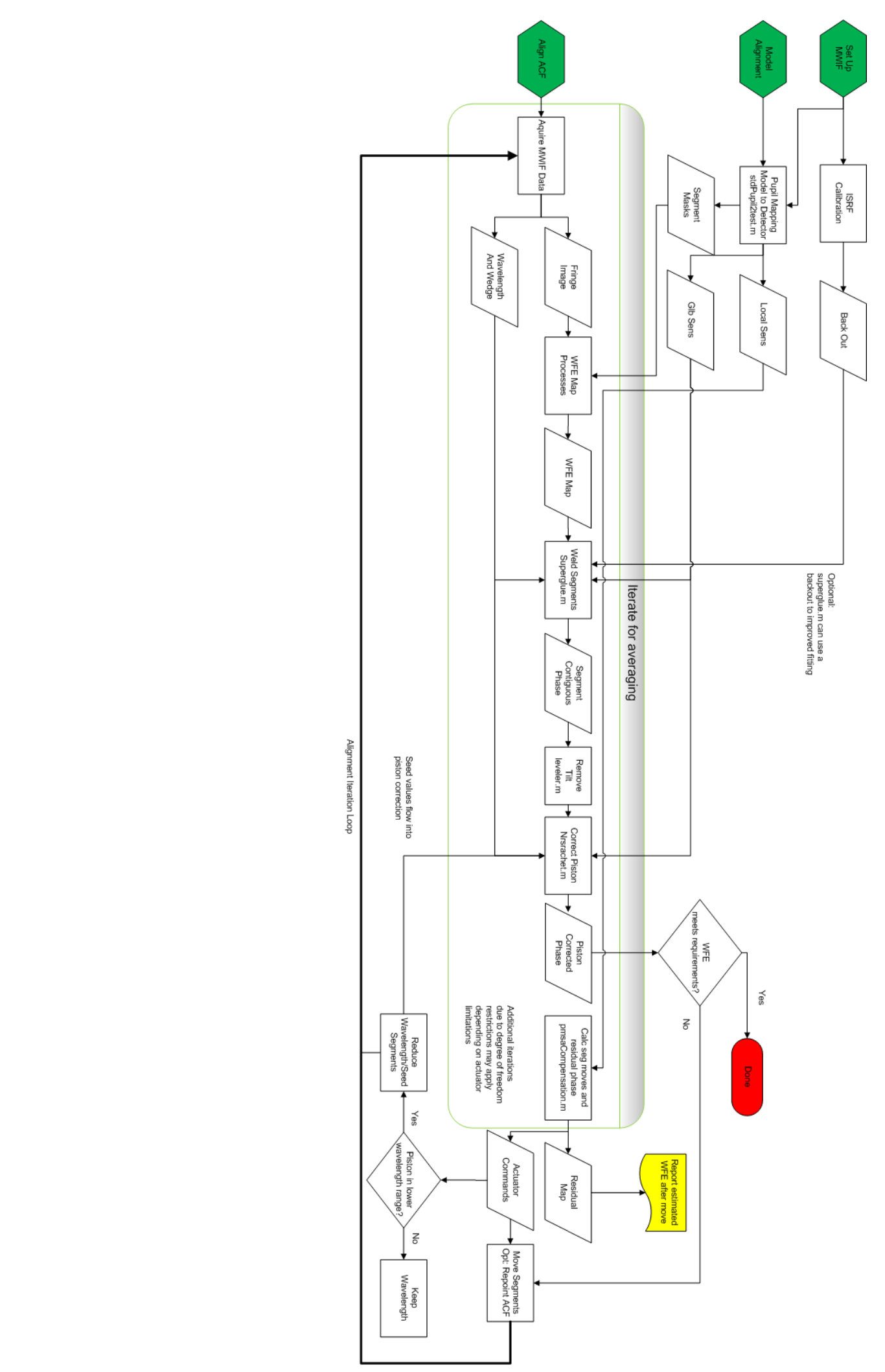

Figure 2. Simplified SASS TBT data analysis flow chart.

22 CFR 125.4 (b)(13) applicable. 


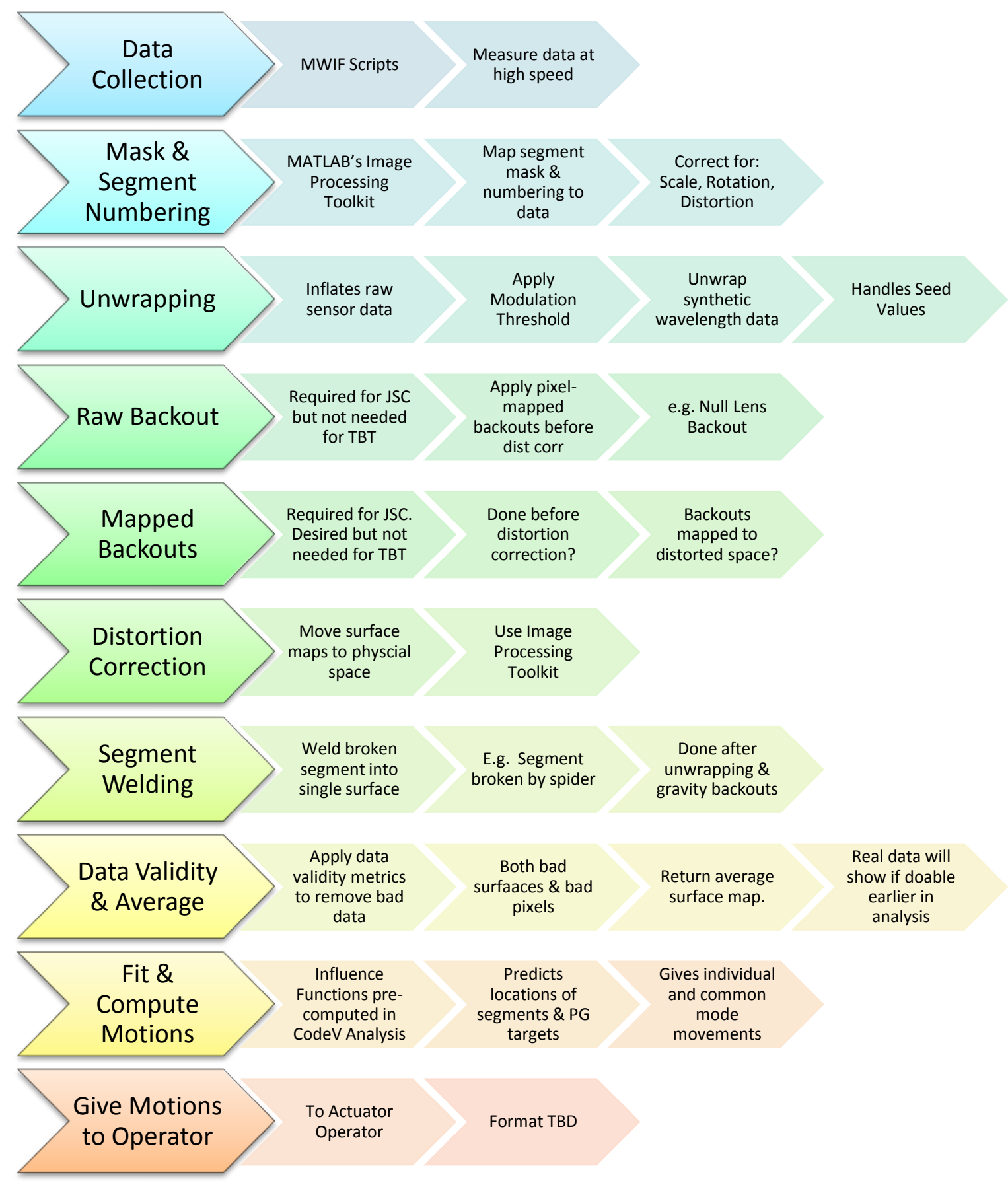

Figure 3. Major Components of the SASS. 


\section{MULTIPLE WAVELENGTH ANALYSIS OF A SEGMENTS SYSTEM}

Much of what makes the MWIF unique is the dual wavelength measurement capability. The MWIF has three sources in all, two fixed-wavelength lasers and one tunable wavelength laser, of which two are used at a time for a single syntheticwavelength measurement. The MWIF uses a clever timing scheme so that two measurements, at different wavelengths, occur in adjacent sequential image frames so that non-common errors due to vibration are minimized. The data from these two measurements in combination is treated as a synthetic wavelength measurement. (Though other data abstractions are possible, see Reference 2). For a pair of fundamental wavelengths $\lambda_{1}$ and $\lambda_{2}$ the synthetic wavelength $\lambda_{s}$ is given by

$$
\lambda_{s}=\frac{\lambda_{1} \cdot \lambda_{2}}{\left|\lambda_{2}-\lambda_{1}\right|}
$$

and the phase map of $\lambda_{s}$ is determined from the different of the phase in $\lambda_{1}$ and $\lambda_{2}$. This process has the effect of increasing the noise in the measurement compared to the fundamental measurement (see Reference 3 for details). For the MWIF the fundamental wavelengths are $660 \mathrm{~nm}$ and $687 \mathrm{~nm}$, and can measure at eight discrete synthetic wavelengths from $16.8 \mathrm{um}$ to $15 \mathrm{~mm}$, given in Table 1 . During a phasing operation the instrument is set to measurement with the longest synthetic wavelength first in expectation of large piston errors. In all cases wavefront tilt has to be in the capture range of the fundamental wavelengths (typically held to less than 100 fringes across the pupil). The PMSAs are then adjusted until the piston of all segments can be unambiguously determined at a shorter available wavelength.

\begin{tabular}{ccrr}
$\begin{array}{c}\lambda 1 \\
(\mathrm{~nm})\end{array}$ & $\begin{array}{c}\lambda 2 \\
(\mathrm{~nm})\end{array}$ & \multicolumn{1}{c}{$\begin{array}{c}\lambda \mathrm{s} \\
(\mathrm{nm})\end{array}$} \\
\hline 687.14 & & & 687 \\
$687.14+660.02 \Rightarrow$ & 16,700 \\
$687.14+683.24 \Rightarrow$ & 120,000 \\
$687.14+686.20 \Rightarrow$ & 500,000 \\
$687.14+686.67 \Rightarrow$ & $1,000,000$ \\
$687.14+686.96 \Rightarrow$ & $2,500,000$ \\
$687.14+687.08 \Rightarrow$ & $7,500,000$ \\
$687.14+687.09$ & $\Rightarrow$ & $10,000,000$ \\
$687.14+687.10 \Rightarrow$ & $15,000,000$
\end{tabular}

Table 1. Absolute and approximate synthetic wavelengths used in the MWIF.

The dynamic range of the MWIF with regard to measuring segment step heights is $\pm \lambda / 4$ for any wavelength (heights are nominally in a direction normal to the surface at the center of each segment i.e. the local $\mathrm{z}$ axis). This is a result of the phase wrapping that occurs for a $2 \pi$ radian change in wavefront. This is often referred to as a " $2 \pi$ ambiguity". Since there is a 2 to 1 relation between surface and wavefront this ambiguity occurs for a $\lambda / 2$ change in height. This is illustrated in Figure 4 below where the second and third fringe orders cannot be distinguished from the zero order fringe. The resolution of this wrapping problem with a synthetic wavelength is depicted in Figure 5. 


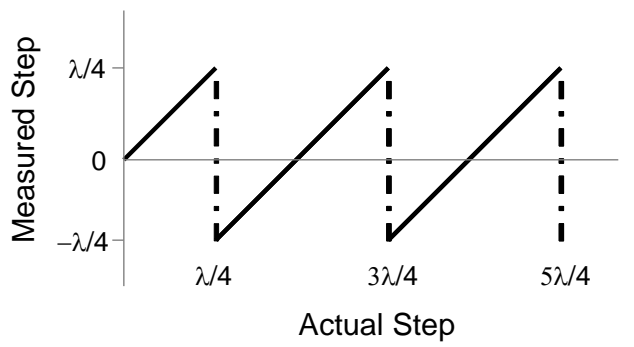

Figure 4. An illustration of step-measurement phase wrapping.

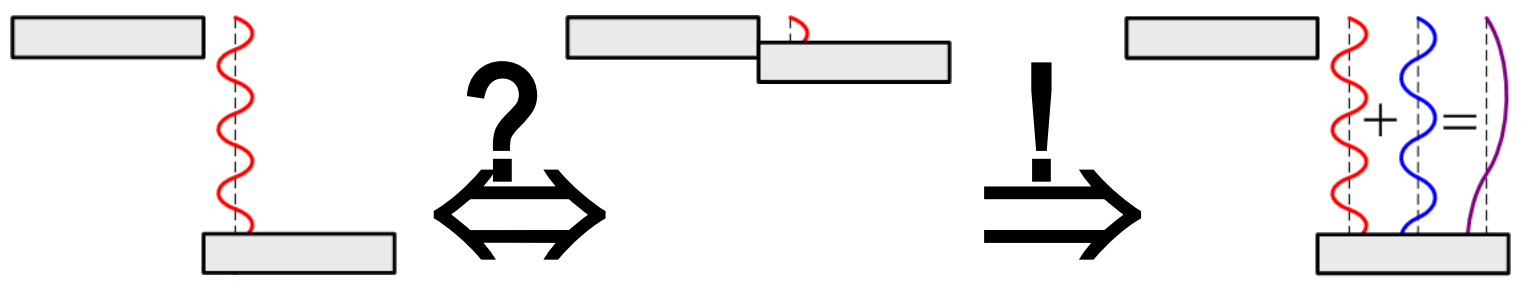

Figure 5. Segment gaps spanning multiples of a shorter wavelength can be resolved by measuring at two wavelengths to create a longer synthetic wavelength.

However, if there is no a priori knowledge of whether a segment is higher or lower than its neighbors then an error may result because the piston offset in the measurement is uncontrolled. This is illustrated in Figure 6. Here piston is adjusted so that one of the three surfaces is set to zero average piston. In two of the three cases the results are erroneous. In general this can be avoided if the total range of the heights is less than $1 / 4$ of the measurement wavelength (which is reduced to $1 / 8$ for the TBT, since its wedge factor is doubled compared to a center of curvature test).

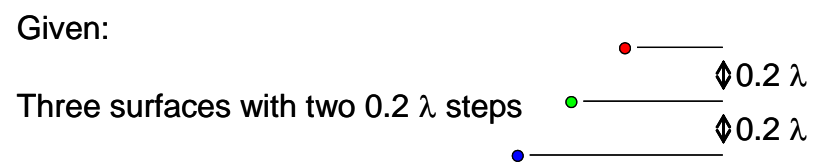

Case 1: Choose to zero $\quad$ Case 2: Choose to zero॰ Case 3: Choose to zero॰

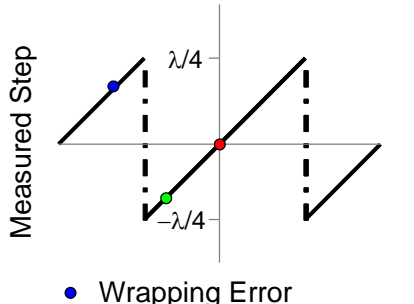

- Wrapping Error

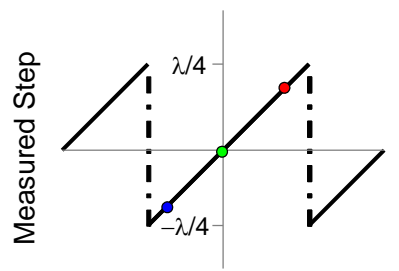

No Wrapping Error

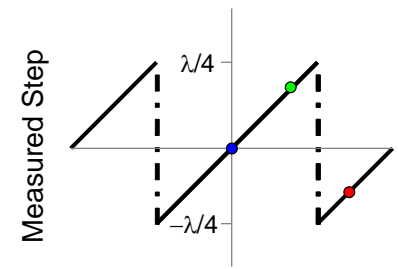

- Wrapping Error

Figure 6. An illustration of correctly choosing the correct unwrapping of adjacent surfaces.

In addition to potential data inversion, the analysis process must consider that there are differences in noise levels at different wavelengths and that biases may be present between different wavelengths.

Consider the case when stepping down from a larger wavelength $\lambda+$ to a shorter wavelength $\lambda-$. As part of the stepdown process one might ask: Can the longer wavelength data confirm that the shorter wavelength data does not suffer a wrapping error? Or if wrapping does occur, is the wrapping well understood and predictable? An illustration of the situation is illustrated in Figure 7. The blue dots represent the sample mean value of height $h_{m}$ as measured at $\lambda$-and its replicants (fringe order). The red dot represents the sample mean value of $h_{m}$ as measured at $\lambda+$. Error bars are shown for an interval of $4 \sigma$ (four standard deviations). 


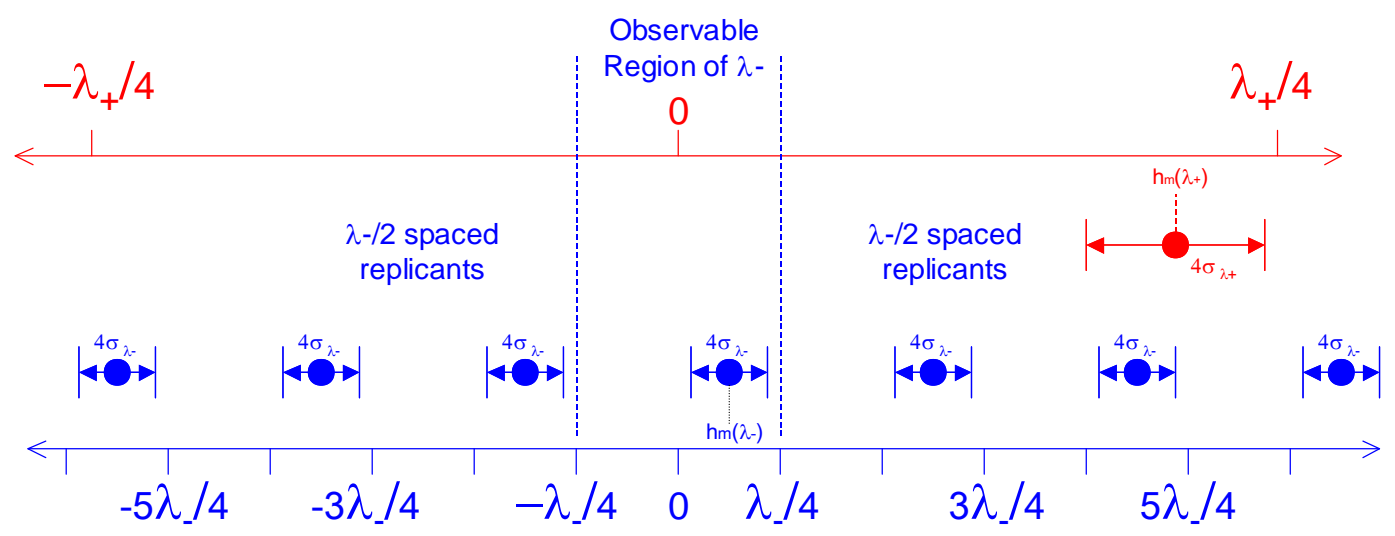

Figure 7. Illustration of step down uncertainty and bias.

In practice when adjusting the piston values between segments the SASS de-ratcheting function (so called because removing the up-is-down errors due to wrapping is implicit to the process) minimizes the error function in Equation (2).

$$
E\left(\text { Bias }_{\text {Fit }}\right)=\sqrt{\sum_{i=1}^{18}\left[\operatorname{Seed}_{i}-\left(k_{i} \frac{\lambda}{2}+\operatorname{wrap}\left(h_{i}-\text { Bias }_{F i t}\right)\right)\right]^{2}}
$$

where $i$ is the index for the segment number, Seed $_{i}$ is the expected values for the height (usually taken from $\lambda+$ data), $_{i}$ is a fringe order guess for the $i^{\text {th }}$ segment, wrap is the sawtooth wrapping function depicted in figure 5 and $h_{i}$ is the raw measured height at $\lambda$-.

Bias $_{F i t}$ is a common offset that is applies to all data and is adjusted during optimization.

\section{POSE ESTIMATION}

The primary use of the JSC center of curvature test is to provide mirror alignment analysis. Given a map that has been processed through the SASS the final step is to estimate the mirror poses using singular value decomposition (SVD) such that a vector $x_{i}$ contains the current alignment estimate for all available degrees of freedom of each $i$ th segment is

$$
x_{i} \quad S_{i}{ }^{1} \times P_{i}
$$

where $S_{i}{ }^{1}$ is the SVD estimated inverse of the sensitivity matrix of the DOFs for the $i$ th segment and $P_{i}$ is the samples pupil data. As mentioned in the Data Flow Overview section, the SASS uses global coordinate and local coordinate versions of $S_{i}{ }^{1}$.

The pose estimation technique is also used inside other components of the SASS. For example: the phase maps of some of the segments are interrupted by the secondary mirror spider shadow. In this case, the phase maps of either side of the shadow need to be reconciled such that $2 \pi$ ambiguities are removed (this occurs prior to segment de-ratcheting which was discussed in the previous section). The method used in the SASS is to discretely vary the phase between the two sections of the broken segment image and use SVD to estimate a theoretical pose of the potential solutions. The solution with the lowest fit error residual is taken as the correct solution. This method is robust compared to edge height matching given the figure error and edge slope discontinuities that are present in the gravity deformed state of the JWST PMSAs during the JSC integration (though it does require knowledge of the system alignment behavior) especially when these deformed states are predictable.

Since pose estimation occurs in the processing loop, it is possible to create statistical run charts and data variability summaries for all segment alignment DOFs. Examples of there are depicted in Figure 8 and Figure 9. 


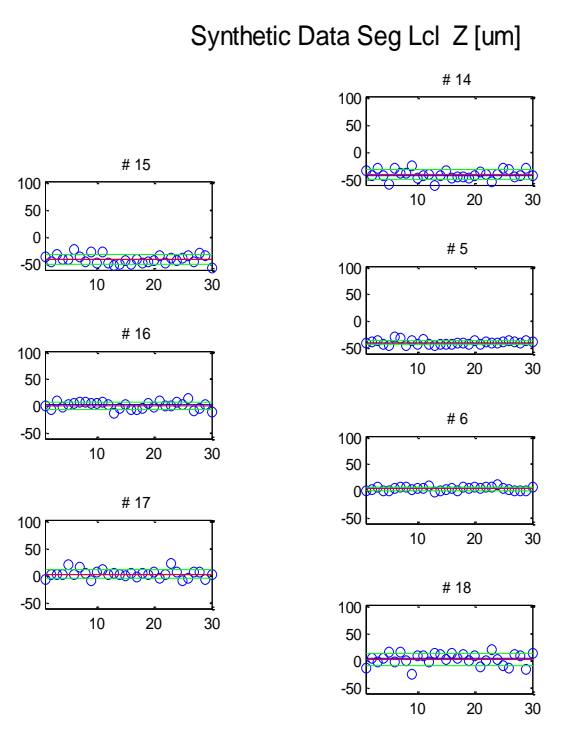

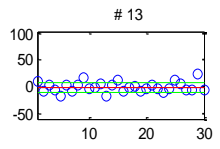
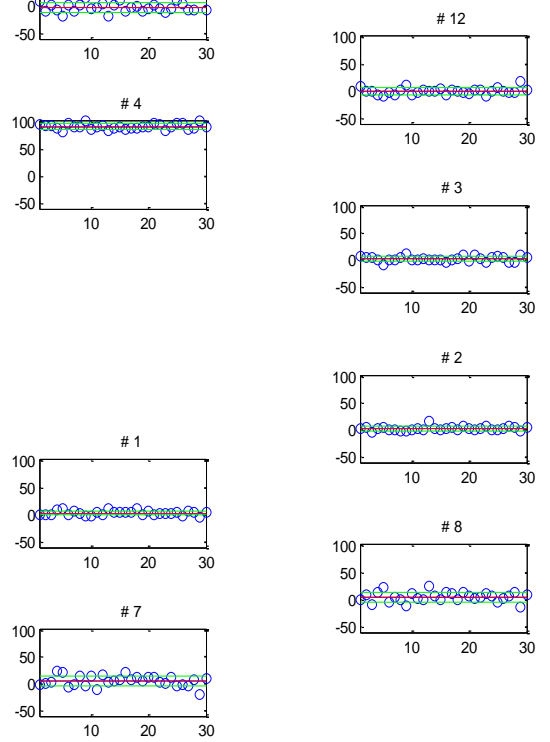
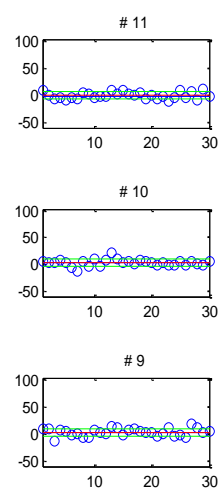

Figure 8. SASS alignment run charts for measured local $\mathrm{z}$ axis displacement of each of the 18 segments. These charts show results taken from a $1 \mathrm{~mm}$ synthetic wavelength analysis of the MWIF data.
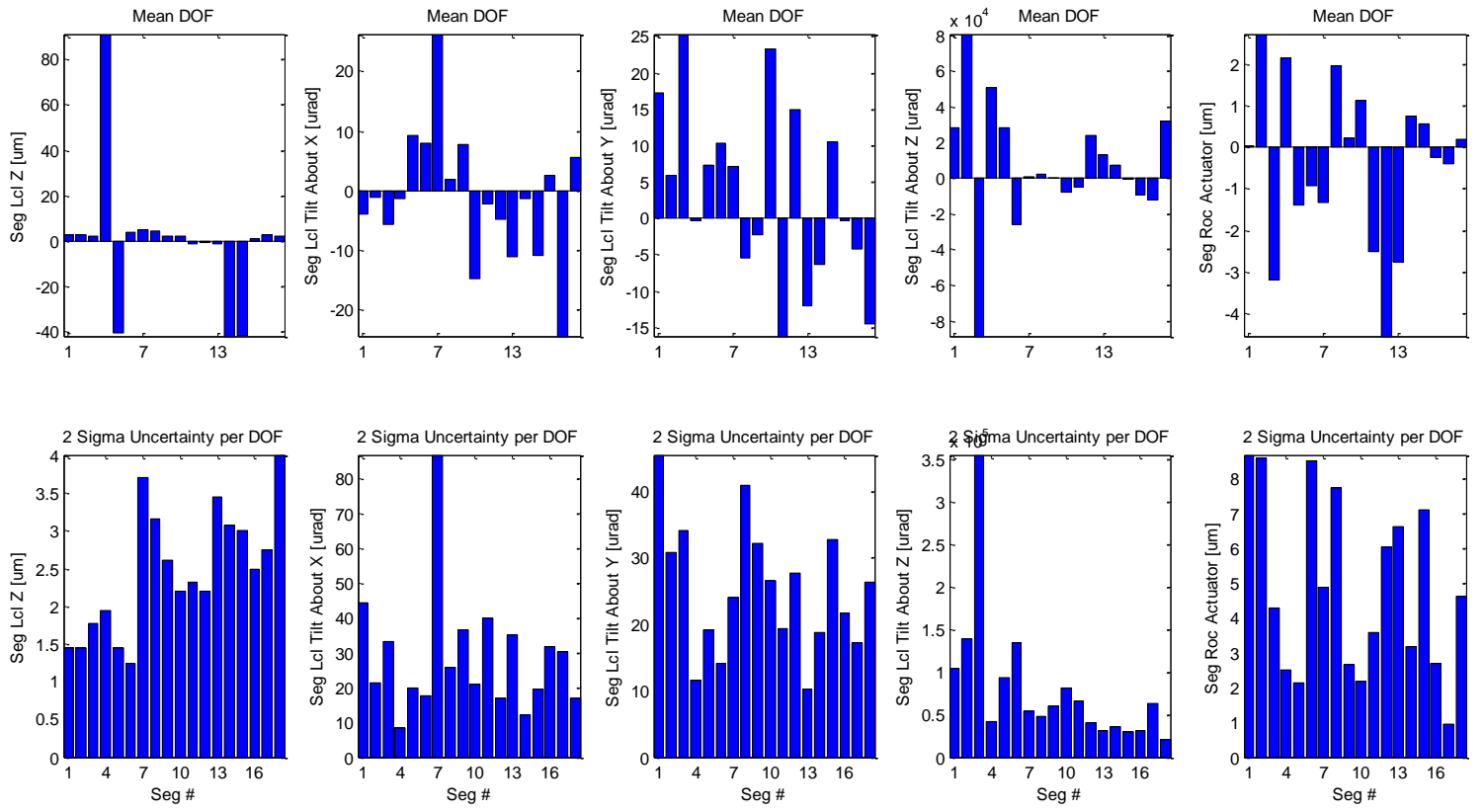

Figure 9. SASS alignmentssummary charts for a 5 DOF analysis for each of 18 segments of the TBT taken from a $1 \mathrm{~mm}$ synthetic wavelength analysis of the MWIF data.

\section{TEST RESULTS}

Because the afocal relay that coupled the MWIF was designed with sub optimal off-the-shelf components it was important to characterize the system up to the insertion point at the TBT. This was accomplished with an auxiliary reference sphere. As shown in Figure 10, there is a large power (and some tilt) bias in the $16.8 \mu \mathrm{m}$ wavelength compared to the fundamental wavelength. This bias is removed with a wavelength-dependent calibration map. 


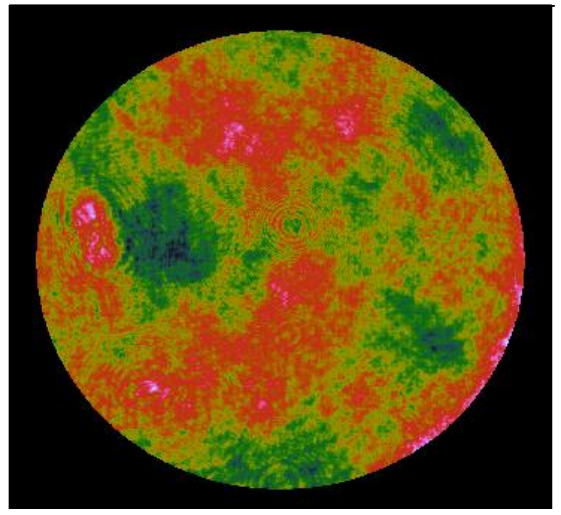

(a) $687 \mathrm{~nm}$
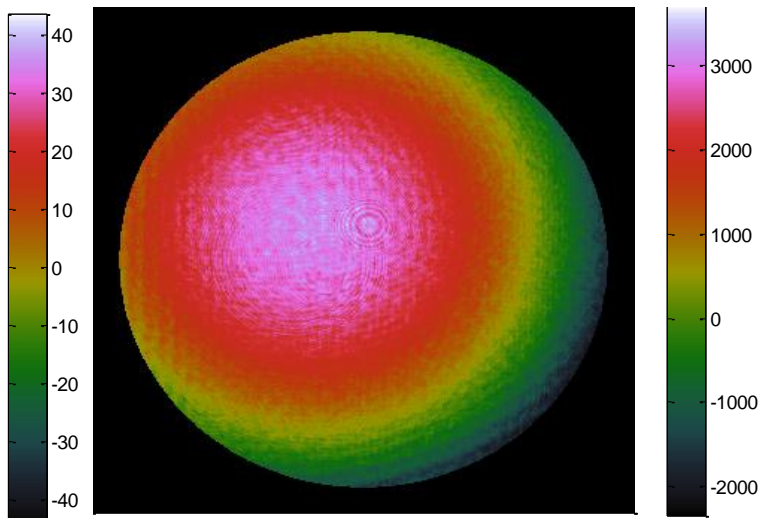

b) $16.8 \mathrm{um}$

Figure 10. TBT insertion MWIF wavefront calibration data and fundamental (687 nm) and synthetic (16.8 um) wavelengths (scale in $\mathrm{nm}$ ).

Once the calibration and pupil mapping data is in place the system is self-checked by running the reference sphere data through the SASS as if it were the actual TBT. The plot in Figure 11 shows the $16.8 \mu \mathrm{m}$ and $687 \mathrm{~nm}$ data for a 100 measurement run (the $687 \mathrm{~nm}$ is usually chosen for comparison since it will be the wavelength for which the JSC test will have null calibration data). Here one can see that the biases between the synthetic (Seed) and fundamental data points is less than $45 \mathrm{~nm}$ for all segments; this is an acceptable level as it is small compared to the fundamental measurement range. The seed value is taken from the average segment piston value for all 100 measurements. This check was done with the reference sphere near null (few tilt fringes). When tilt fringes are introduced the results are less favorable. There are significant differential fringe print-through artifacts that add bias to the synthetic wavelength data. This effect is depicted in Figure 12 (power and tilt are removed from the map). The peak-to-valley error of this effect has been observed at values as high as $3 \mu \mathrm{m}$; in the present of such an error the fundamental wavelength fringe order determination can produce erroneous results. Test results show that the effect can be mitigated if the reference mirror is displaced alone the optical axis a distance of $1 / 2$ of the synthetic wavelength during a measurement sequence.

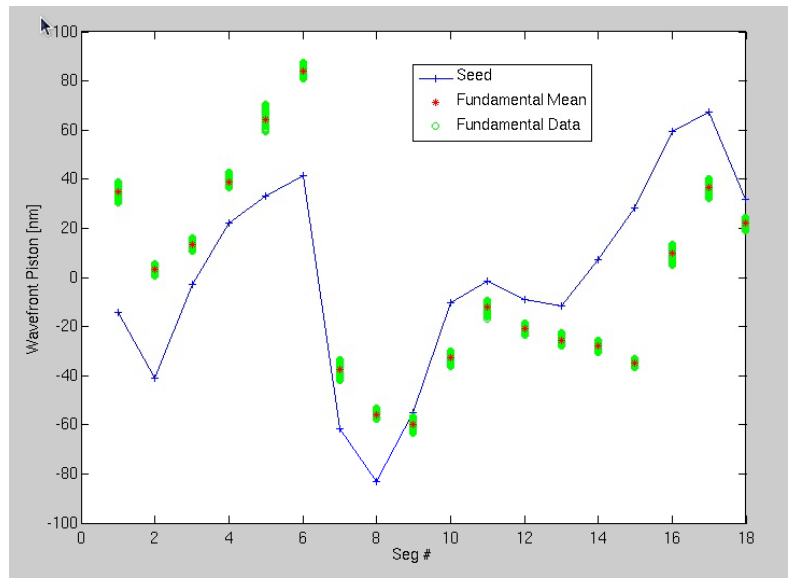

Figure 11. SASS self check of TBT analysis. 


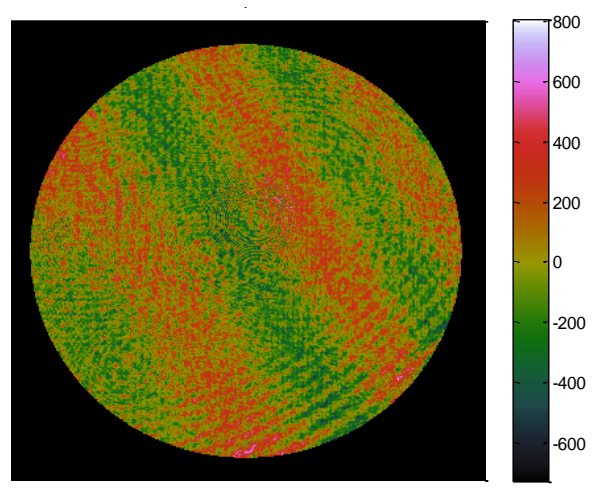

Figure 12. Synthetic wavelength data for a reference sphere with tilt fringes (wavefront power and tilt removed). Diagonal fringe print-through errors are observable in the map.

Pupil maps for segment phasing trial are shown in Figure 13. Here the segments are initially randomly phased so the peak-to-valley wavefront error is $2 \mathrm{~mm}$. After six segment alignment adjustments the root mean square (rms) piston is reduced to $54 \mathrm{~nm}$. Further improvement would have been possible but for some control problems with segment 4 (the inner segment at 12 o'clock in the figure).
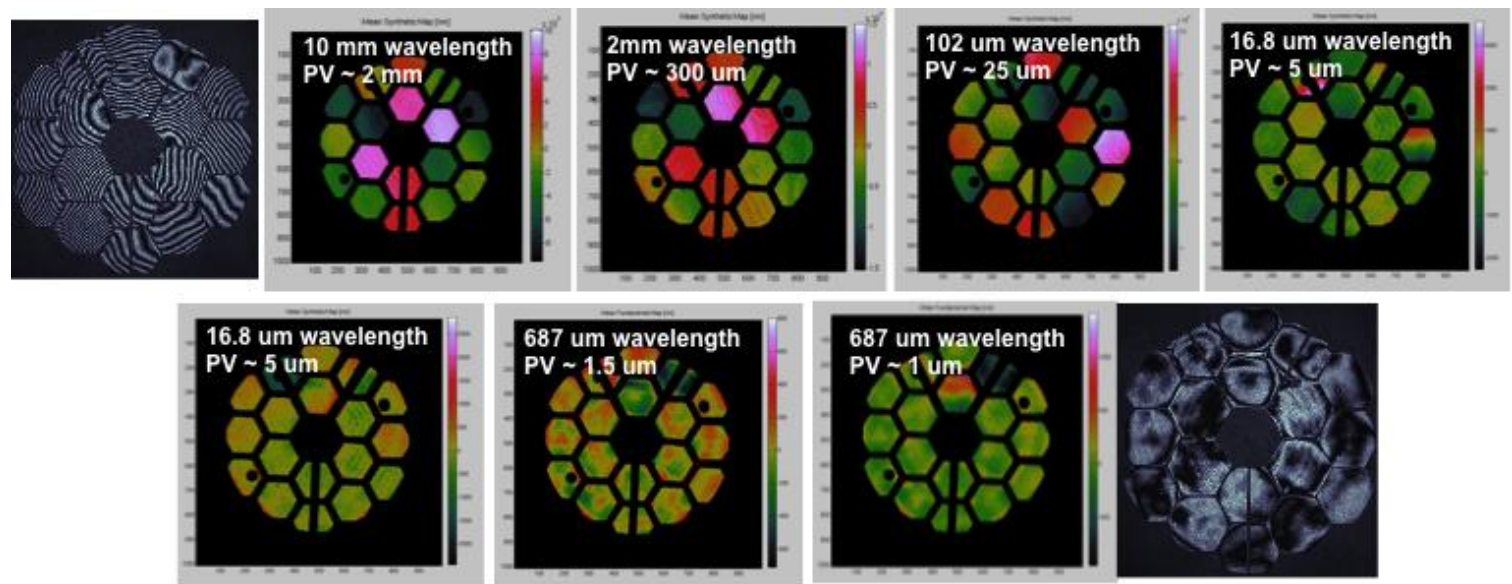

Rms surface piston $=54 \mathrm{~nm}$

Figure 13. Pupil maps for each step of a TBT phasing experiment.

An additional analysis feature of the SASS is separating out and reporting the global error of PM with respect to the center of curvature null lens. Global errors are estimated from individual segment position error estimates using an alignment model where each segment is a common coordinate system (e.g. the parent vertex). In this case, the mean of each rigid body motion provides the common mode error in the position of the segments. Removing the global errors is important since these errors will drift as a function of the position of the metrology system relative to the telescope without providing useful PM alignment information

Global alignment error was simulated in the TBT by intentionally decentering the secondary mirror to induce wavefront coma at the MWIF field point (which normally has very little coma). The results are shown in Figure 14. The predicted global PM alignment is consistent with the compensation required for the secondary mirror misalignment used in the simulation. 


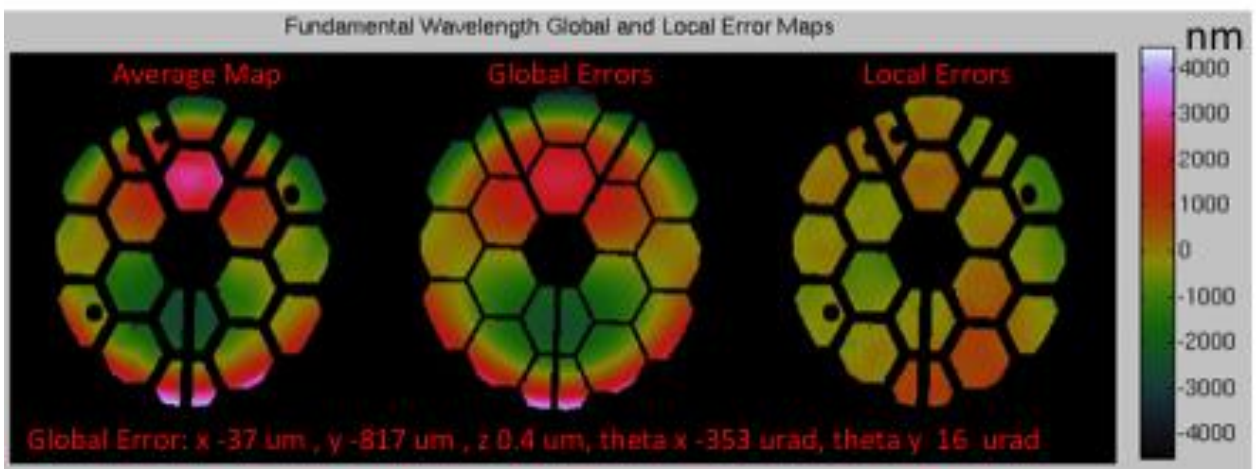

Figure 14. Global alignment estimation for a comatic pupil.

\section{CONCLUSIONS}

We have demonstrated the ability to phase the TBT 18 segment primary mirror using the 4D Technology MWIF and ITT's CASS software to approximately $54 \mathrm{~nm}$ rms surface error. These demonstrate that the MWIF will be capable of meeting the requirements of JWST integration at JSC.

\section{ACKNOWLEDGEMENTS}

Scott Acton (Ball Aerospace), Doug Leviton (NASA Goddard Space Flight Center), Tony Whitman (ITT), Mark Waldman (Sigma Space)

\section{REFERENCES}

[1] North-Morris, Michael B., Millerd, James E., Brock, Neal J. and Hayes, John B., "Phase-shifting multiwavelength dynamic interferometer", Proc. SPIE 5531, 64 (2004).

[2] Decker, Jennifer E. Miles, John R., Madej, Alan A., Siemsen, Ralph F., Siemsen, Klaus J., de Bonth, Sebastian, Bustraan, Krijn, Temple, Sara and Pekelsky, James R., "Increasing the range of unambiguity in step-height measurement with multiple-wavelength interferometry-application to absolute long gauge block measurement", Appl. Opt. 42, No. 28 (2003).

[3] Zhang, Yan, Redding, David C. and Basinger, Scott A., "Models of Multiple Wavelength Phase Camera (MWPC) for JWST Ground Alignment", Final Report, (Version 1.1), Jet Propulsion Laboratory, Pasadena CA, October 20, 2005. 